\title{
Lead(II) fluoride particles synthesized by a straightforward mechanochemical
}

route.

Maria Angels Subirana-Manzanares, ${ }^{a}$ Marta Sanchez-Sala, ${ }^{a}$ Josefina Pons, $^{a}$ Concepción Domingo ${ }^{b}$ and José A. Ayllón ${ }^{a, *}$

${ }^{a}$ Departament de Química, Universitat Autònoma de Barcelona, Campus UAB, 08193 Bellaterra, Spain.

${ }^{b}$ Institut de Ciència dels Materials de Barcelona (CSIC), Campus UAB, 08193 Bellaterra, Spain.

* Corresponding author

E-mails: joseantonio.ayllon@uab.es.

Tel: +34 93581 2176, Fax: +34 935812920 .

\section{Keywords:}

Mechanochemical; Lead(II) fluoride; Low temperature; Crystalline phase

\begin{abstract}
A low temperature mechanochemistry procedure was used to prepare lead(II) fluoride particles, with the objective of synthetizing pure $\alpha$ and $\beta$ phases. Lead acetate trihydrate was reacted with ammonium fluoride by hand-grounding, thus forming a white liquid mixture containing lead(II) fluoride, water and ammonium acetate. A further short thermal treatment, performed at a low temperature $\left(\mathrm{ca} .100{ }^{\circ} \mathrm{C}\right)$, allowed the evaporation of by-products, giving directly a powder constituted by pure $\mathrm{PbF}_{2}$. According to XRD analysis, the obtained product involved mostly the cubic $\beta-\mathrm{PbF}_{2}$ phase, with only traces of the orthorhombic $\alpha-\mathrm{PbF}_{2}$ phase. SEM analysis indicated the formation of crystalline aggregates, without a well defined morphology. This synthetic strategy could be easily modified by adding small quantities of organic additives used to tune the crystalline phase. Hence, the addition of either citric acid or poly(acrylic acid-co-maleic acid) facilitated the production of the cubic phase $\left(\beta-\mathrm{PbF}_{2}\right)$, while the addition of selected amines strongly favored the crystallization of the orthorhombic phase $\left(\alpha-\mathrm{PbF}_{2}\right)$.
\end{abstract}




\section{Introduction}

Mechanochemistry, a synthesis method known for a long time [1], has had a limited use to data. However, the interest in mechanochemical processes has recently increased, mainly due to the recognized advantages of this technique in regard of the enhanced energy, time and resources use efficiency [2,3]. Hence, mechanochemistry is currently being developed by researchers as a sustainable alternative synthesis technique, which is primarily due to the change in the way chemists approach the use of solvents by taking into account the concepts of green chemistry.

Designed mechanochemical processes are not always totally solvent-free, since they can also involve a small amounts of solvent, contained either in the reagents or added intentionally. Indeed, solvent (or liquid) assisted mechanochemistry has greatly enhanced the applicability of this synthetic method, while reducing the time of materials preparation. Moreover, the added liquid can be used as a supplementary experimental parameter to determine the chemical nature of the final product [3].

Mechanochemistry allows for the synthesis of a great variety of compounds and materials, including organics and inorganics, and also complex composites and hybrids. [2,4] The diverse practical applications of mechanochemistry involve ceramics processing, mechanical alloying, organic and inorganic synthesis and polymer technology. This work addresses the mecanochemical synthesis of $\mathrm{PbF}_{2}$, with special emphasis in the preparation of pure phases. The process is assisted by a low-temperature post-treatment procedure to eliminate by-products. Only few examples of other metal fluorides $\left(\mathrm{BaF}_{2}\right.$, $\mathrm{SrF}_{2}$ and $\mathrm{CaF}_{2}$ ) obtained by mechanochemistry can be found in the literature, which were obtained "just by shaking” together the corresponding hydroxides and $\mathrm{NH}_{4} \mathrm{~F}$ used as a fluorinating agent [5]. Different metal precursors, such as carbonates, acetates or formates, have also been assessed to synthetize these fluorides, but the mixtures obtained with $\mathrm{NH}_{4} \mathrm{~F}$ required vigorous and energetic ball milling to form the desired products. Details about elimination of by-product are not given [5,6].

$\mathrm{PbF}_{2}$ is found in two distinct crystalline phases: $\beta-\mathrm{PbF}_{2}$ with a cubic fluorite structure and $\alpha-\mathrm{PbF}_{2}$ with an orthorhombic structure [7]. The transition between the two phases is complex, and even some controversy is still established in regard of which is the most stable phase at ambient conditions [8-10]. Both orthorhombic and cubic phases and also their mixtures can be obtained at low temperature depending on the processing route. Moreover, it has been reported that at room temperature $\beta-\mathrm{PbF}_{2}$ transforms to $\alpha$ $\mathrm{PbF}_{2}$ just by immersion in water for several hours [11] or by adding an excess of fluoride anion in a $\beta$ $\mathrm{PbF}_{2}$ methanol solution [12]. 
$\mathrm{PbF}_{2}$ is a highly technological material with different proposed applications, essentially when it is presented in a single ( $\alpha$ or $\beta$ ) crystalline phase [13]. For example, superionic conductivity has been described for the $\beta$-phase [10] and also it has been used as a host for $\mathrm{Dy}^{3+}$ ions to enhance their quantum efficiency upon UV excitation [14]. $\alpha-\mathrm{PbF}_{2}$ is a strong scintillator that can be used as a detector in an electromagnetic calorimeter [11]. Hence, the design of synthetic strategies that lead to the formation of a single $\mathrm{PbF}_{2}$ crytalline phase is of a great interest.

Several routes for the synthesis of $\mathrm{PbF}_{2}$ have been reported. In solid state, the formation by thermolysis of $\mathrm{Pb}(\mathrm{II})$ precursors of both macroscopic crystals and nanoparticulated cubic $\beta-\mathrm{PbF}_{2}$ has been reported [9]. However, to obtain the needed precursors containing fluorinated ligands, an extremely laborious synthesis is required. A physical route, based on the condensation of precursors in an inert gas, has been described to produce nanostructured $\mathrm{PbF}_{2}$, but it requires an ultra-high vacuum equipment [10]. Two publications account for the use of lead(II) acetate and ammonium fluoride, both common commercial reagents, to prepare $\mathrm{PbF}_{2}$ The first one use water solution chemistry, being the crystalline phase and porosity of the produced $\mathrm{PbF}_{2}$ microparticles determined by the use of additives [15]. In the second, methanol solution chemistry with ultrasounds activation is used to obtain, octahedron-like $\alpha-\mathrm{PbF}_{2}$ microparticles [16]. In this work, after a deep study of the best mechanochemistry conditions for $\mathrm{PbF}_{2}$ synthesis, several carboxylic acids and amines, with different sizes and functional groups, were assayed as additives to control the crystalline structure. The powdered material produced was structural and morphologically characterized. Experimental conditions to obtain near pure phase materials were established.

\section{Experimental}

\subsection{Materials}

Lead(II) acetate trihydrate $\left(\mathrm{Pb}\left(\mathrm{CH}_{3} \mathrm{COO}\right)_{2} \cdot 3 \mathrm{H}_{2} \mathrm{O}\right)$ and ammonium fluoride $\left(\mathrm{NH}_{4} \mathrm{~F}\right)$ were used to synthetize $\mathrm{PbF}_{2}$. Phenylvaleric, 3,6,9-trioxadecanoic acid, benzoic, tartaric and citric acids, the polymer poly(acrylic acid-co-maleic acid) (PACMA, 50 \%wt water solution) and the amines dodecylamine $\left(12 \mathrm{NH}_{2}\right)$ and 2-amino-2-methyl-1-propanol (AMPOH) were used as additives. All reagents were purchased from Sigma-Aldrich and used without further purification.

\subsection{Synthetic procedure}


In a reference synthesis, $\mathrm{Pb}\left(\mathrm{CH}_{3} \mathrm{COO}\right)_{2} \cdot 3 \mathrm{H}_{2} \mathrm{O}(1.00 \mathrm{~g}, 2.66 \mathrm{mmol})$ and $\mathrm{NH}_{4} \mathrm{~F}(0.200 \mathrm{~g} 5.35 \mathrm{mmol})$ were hand-grounded using a Teflon mortar and pestle. On grounding, the reagents mixture quickly acquired a liquid texture, which favored homogenization. At this point, the obtained white mixture was kept in a furnace with forced ventilation (air) pre-heated at $100{ }^{\circ} \mathrm{C}$ for a short period of time (1-4 h), until the measurement of constant weight for the sample indicated complete elimination of volatile by-products. Recovered product was considered as the reference material. In further experiments, a small amount of one of the studied organic additives was first grounded with $\mathrm{Pb}\left(\mathrm{CH}_{3} \mathrm{COO}\right)_{2} \cdot 3 \mathrm{H}_{2} \mathrm{O}$ before the addition of $\mathrm{NH}_{4} \mathrm{~F}$. Either a 10:1 or a 6:1 lead:additive molar ratio was employed for the studied carboxylic acids or amines, respectively. For the the polymer PACMA, a weight ratio lead acetate:additive aqueous solution of 10:1 was used. Note that the PACMA product is only available in a $50 \%$ wt water solution, therefore, its use entails the addition of some more water $(0.050 \mathrm{~g})$ to the initial precursor mixture. However, this amount of water can be considered as negligible when compared to the $0.142 \mathrm{~g}$ of water contained in the $1.00 \mathrm{~g}$ of $\mathrm{Pb}\left(\mathrm{CH}_{3} \mathrm{COO}\right)_{2} \cdot 3 \mathrm{H}_{2} \mathrm{O}$ reagent. In all the experiments, a white solid residue was formed and recovered for characterization. No further cleaning was necessary.

\section{Results and discussion}

The used mechanochemical synthesis was a solvent-assisted process, as indicated by the liquid texture of the mixture obtained by grounding the precursors. The liquid source was the crystallization water enclosed in the lead acetate trihydrate reagent, which corresponds to a $12 \%$ wt of the precursor mixture. In some mechanochemistry processes, heat is applied after synthesis to favor the elimination of labile ligands $[17,18]$. In this study, a low temperature heating procedure $\left(100^{\circ} \mathrm{C}, 1 \mathrm{~h}\right)$ was applied after mechanochemical synthesis to evaporate water and to sublimate ammonium acetate by-products [19]. This procedure yielded directly $\mathrm{PbF}_{2}$ as a dry and solvent-free material from available commercial precursors. Moreover, the reduced diffusion of reagents in the low-solvent content, low-temperature medium limited crystal growth yielding submicron particles. After by products elimination, the measured final weight was in accordance with the stoichiometric formation of lead(II) fluoride in the reference sample. XRD analysis confirmed the formation of this product (Fig. 1(a)). The cubic $\beta-\mathrm{PbF}_{2}$ phase was mainly formed following the reference synthesis procedure, i.e. without additive addition, in which only traces of the orthorhombic phase were observed (arrows in the spectrum of Fig 1(a)). 
Once the wellness of the mechanochemical route to obtain $\mathrm{PbF}_{2}$ was established, main efforts were directed to study the effect of additives on the crystalline phase. The addition of capping ligands is a common tool used to control the properties of nanoparticles [20,21]. Thus, carboxylate anions have previously been used to stabilize rare earth/lantanide fluoride nanoparticles [22,23]. In this work, mono-, di- and tricarboxilic acids, as well as the acid polymer PACMA, were used to analyze the effect of additives on the crystalline phase, purity and particle morphology. The addition of dicarboxylic tartaric acid yielded essentially the cubic $\beta-\mathrm{PbF}_{2}$ phase, with only traces of $\alpha$-phase (Fig. S1, see Supplementary Material), while the addition of either the tricarboxylic citric acid or the PACMA allowed to obtain pure $\beta$-phase (Fig. 1(b)).Contrarily, the addition of monodentate carboxylic acids led to the formation of mixtures of $\beta$ and $\alpha$ phases (Fig. S2, see Supplementary Material), which not the objective of this study, since these mixtmres have not yet described applications. Yo esto se lo excribiria al referee pero no lo añadiria. Following the described mechanochemical procedure, the use of amines as additive strongly favored the crystallization of $\alpha-\mathrm{PbF}_{2}$ (Fig.1(c)), even though small traces of the cubic $\beta$-phase were also detected in the XRD analysis.

In short, for samples obtained from $\mathrm{Pb}$ (acetate) $)_{2}$ and $\mathrm{NH}_{4} \mathrm{~F}$ without any additive all diffraction peaks with a significant intensity could be perfectly indexed to the $\beta-\mathrm{PbF}_{2}$, in agreement with the literature values in JCPDS $n^{\circ} 06-251$, and only minor peaks assignable to the $\alpha-\mathrm{PbF}_{2}$ (JCDPS $\left.n^{\circ} 41-1086\right)$. For all this samples, the amount of second phase impurity was very small, as reflected by the low intensity in the trace peaks (arrows in Fig. 1(c)). Using citric acid or poly(acrylic acid-co-maleic acid) the traces of the $\alpha$ phase did not appear, and only pure $\beta$-phase was observed. For samples obtained from $\mathrm{Pb}(\text { acetate })_{2}$ and $\mathrm{NH}_{4} \mathrm{~F}$ with an amine, all diffraction peaks with a significant intensity could be perfectly indexed to the $\alpha$ $\mathrm{PbF}_{2}$, in agreement with the literature values in JCDPS nº 41-1086.

\section{Figure 2}

SEM characterization was performed only on samples with the largest degree of crystalline phase purity. For the cubic phase obtained in the reference syntesis, SEM images showed large aggregates of small particles (Fig. 2(a)). The pure cubic phase obtained in the presence of citric acid was constituted of discernible individual submicrometric crystals, forming irregular aggregates (Fig. 2(b)). The material obtained with the addition of PACMA had a similar aspect than the reference material (Fig. 2(c)). The 
orthorhombic $\mathrm{PbF}_{2}$ material obtained in the presence of amines showed larger (micrometric) and better defined crystals than the previous ones. Each amine determined a different morphology for the formed crystals. Thus, the use of $12 \mathrm{NH}_{2}$ yielded isometric polyhedral crystals, (Fig. 2(d)) while the use of AMPOH favored the formation of elongated architectures (Fig. 2(e)). In both cases, nano-holes were observed on the crystal faces, likely formed during the evaporation of ammonium acetate by-product during heating in the performed post-treatment. ) SEM analysis indicated that systems involving amine additives are better crystallized with larger particles than systems prepared without additive addition. On the basis of these experimental results, we suggest that the amines are effective capping agents for the $\mathrm{PbF}_{2}$ nucleated particles forming a shell that surrond them and prevents their agglomeration, thus, favoring crystal growth. As a result, polyhedral large particles are obtained.

\section{Figure 2}

Fig. 3 shows the ATR-FTIR spectra of the reference and additive-addition materials described for SEM analysis. Bands at 1518 and $1425 \mathrm{~cm}^{-1}$ in Fig. 3a denoted the presence of traces of acetate in the reference material. Intense bands assigned to carboxylate were observed in the spectrum of the $\beta-\mathrm{PbF}_{2}$ sample obtained by adding either citric acid or PACMA (Fig 3b,c). In both cases, a less intense band at ca. 1705 $\mathrm{cm}^{-1}$ indicated the presence of minor quantities of protonated carboxylic acid. Finally, spectra in Fig. 3d,e displayed some bands assigned to residual amines, which remained adsorbed in the final product. For the sample prepared using 12NH $\mathrm{N}_{2}$ in Fig. 3d, the two intense bands at 2956 and $2849 \mathrm{~cm}^{-1}$ were assigned to $\mathrm{C}-\mathrm{H}$ stretching, while the weak band at $3230 \mathrm{~cm}^{-1}$ was assigned to $\mathrm{N}-\mathrm{H}$ stretching [24]. The later band was shifted with respect to pure amine $\left(3325 \mathrm{~cm}^{-1}\right)$, indicating that $12 \mathrm{NH}_{2}$ was likely anchored on the $\mathrm{PbF}_{2}$ surface through the $\mathrm{N}$ atom [25].

\section{Figure 3}

\section{Conclusions}

The mechanochemical synthesis of $\mathrm{PbF}_{2}$ from commercial precursors, lead(II) acetate and $\mathrm{NH}_{4} \mathrm{~F}$ as fluorinating agent, was here studied. The water contained in the lead(II) precursor allowed an easy homogenization of the reaction mixture, being hand-grounding an enough energetic process for product 
formation. The use of additives provided control of the crystalline phase in the final product. Hence, the use of polyacids favored the formation of the cubic phase, while the use of amines direct the reaction to the formation of the orthorhombic phase preferentially.

\section{Acknowledgements}

This work was partially financed by the Generalitat de Catalunya 2014SGR377 and by the Spanish National Plan of Research CTQ2014-56324.

\section{References}

[1] Parker, LH. J. Chem. Soc., Trans. 1914;105:1504-16.

[2] James SL, Adams CJ, Bolm C, Braga D, Collier P, Friscic T, et al. Chem. Soc. Rev. 2012;41:413-47.

[3] Bowmaker GA. Chem. Commun. 2013;49:334-48.

[4] Xu C, De S, Balu AM, Ojeda M, Luque R. Chem. Commun. 2015;51:6698-713.

[5] Dreger M, Scholz G, Kemnitz E. Solid State Sci. 2012;14:528-34.

[6] Scholz G, Dörfel I, Heidemann D, Feist M, Stösser R. J. Solid State Chem. 2006;179:1119-28.

[7] Hull S, Keen D. Phys. Rev. B 1998;58:14837-44.

[8] Monteil A, Chaussedent S, Guichaoua D. Mater. Chem. Phys. 2014;146:170-4.

[9] Erk C, Hammerschmidt L, Andrae D, Paulus B, Schlecht S. Phys. Chem. Chem. Phys. 2011;13:602935.

[10] Thangadurai P, Ramasamy S, Manoharan PT. Eur. Phys. J. B 2004;37:425-32.

[11] Alov DL, Rybchenko SI. J. Phys.: Condens. Matter. 1995;7:1475-82.

[12] Huang B, Hong JM, Xue ZL, You XZ. Solid State Commun. 2005;133:393-5.

[13] Plekhanov VG. Prog. Mater. Sci. 2004;49:787-886.

[14] Sarkar S, Hazra C, Chatti M, Sudarsan V, Mahalingam V. RSC Adv. 2012;2:8269-72.

[15] Zhu G, Liu P, Hojamberdiev M, Zhou JP, Huang X. J. Mater. Sci. 2010;45:1846-53.

[16] Xu K. Mater. Lett. 2008;62:4322-4.

[17] Kuroda R, Yoshida J, Nakamura A, Nishikiori SI. CrystEngComm, 2009;11:427-32

[18] Yoshida J, Nishikiori SI, Kuroda R. Chem. Eur. J. 2008;14:10570-8

[19] Chaiken RF, Sibbett DJ, Sutherland JE, Van De Mark DK, Wheeler A. J. Chem. Phys. 1962;37:2311-18.

[20] Estruga M, Domingo C, Ayllón JA. J. Mater. Chem. 2011;21, 4408-15.

[21] Estruga M, Roig A, Domingo C, Ayllón JA. J. Nanoparticle Res. 2012;14:1053.

[22] Li X, Gai S, Li C, Wang D, Niu N, He F, Yang P. Inorg. Chem. 2012;51:3963-71

[23] Chaput F, Lerouge F, Tusseau-Nenez S, Coulon PE, Dujardin C, Denis-Quanquin S, et al. Langmuir 2011;27:5555-61

[24] Renuka NK, Shijina AV, Praveen AK. Mater. Lett. 2012;82:42-4.

[25] Aslam M, Schultz EA, Sun T, Meade T, Dravid VP. Cryst. Growth Design 2007;7:471-5 
Figure captions

Fig. 1. Powder XRD pattern of $\mathrm{PbF}_{2}$ samples: (a) reference material; and products obtained with additives: (b) citric acid or PACMA; and (c) $12 \mathrm{NH}_{2}$ or AMPOH.

Fig. 2. SEM micrographs of $\mathrm{PbF}_{2}$ samples: (a) reference material; and products obtained with additives: (b) citric acid; (c) PACMA; (d) 12NH ; and (e) AMPOH. Bar corresponds to $200 \mathrm{~nm}$.

Fig. 3. ATR-FTIR spectrum of $\mathrm{PbF}_{2}$ samples: (a) reference material; and products obtained with additives: (b) citric acid; (c) PACMA; (d) $12 \mathrm{NH}_{2}$; and (e) AMPOH. 
Figuras articulo $\mathrm{PbF}_{2}$
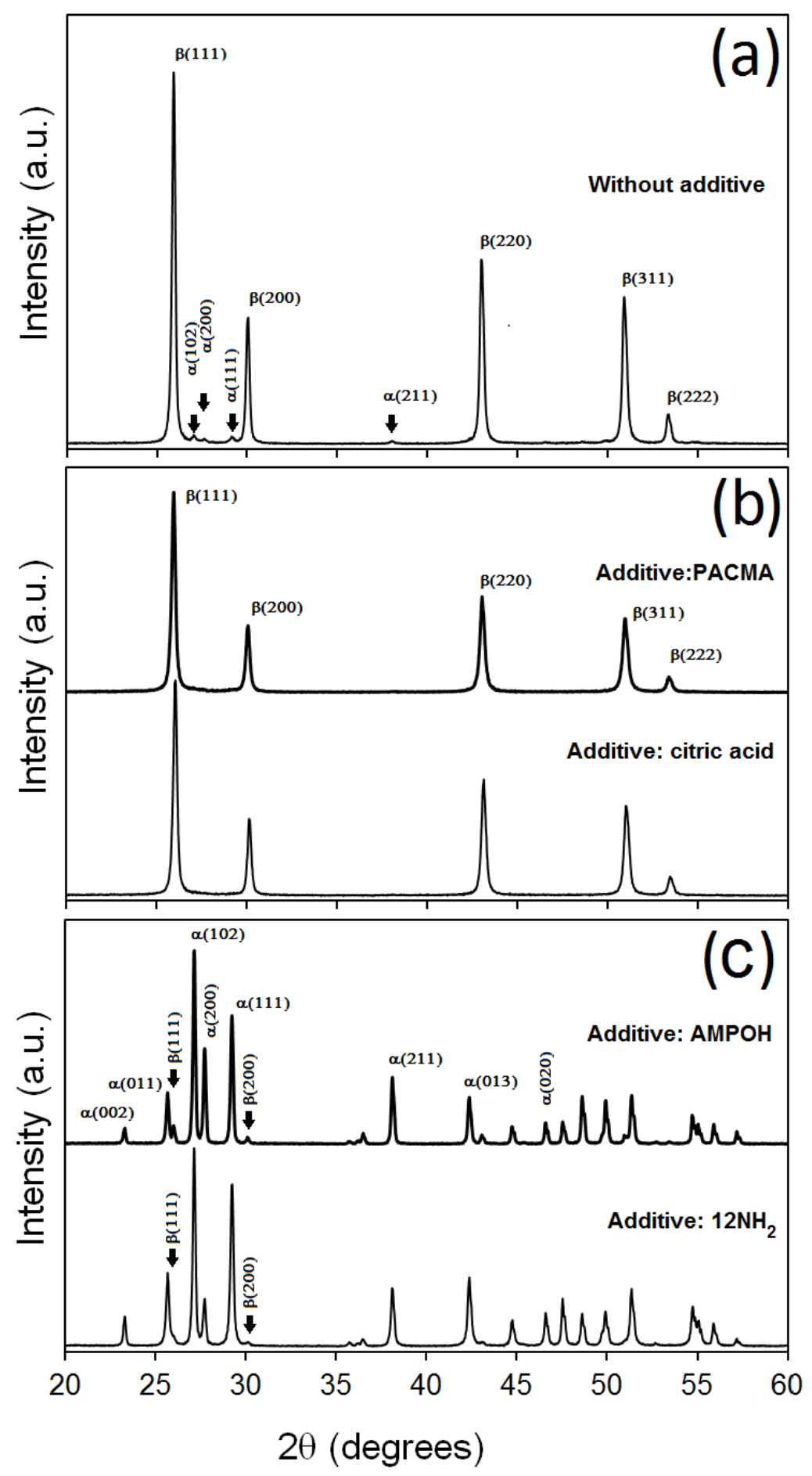

Fig. 1. Powder $\mathrm{XRD}$ pattern of $\mathrm{PbF}_{2}$ samples: (a) reference material; and products obtained with additives: (b) citric acid or PACMA ; and (c) $12 \mathrm{NH}_{2}$ or AMPOH. 

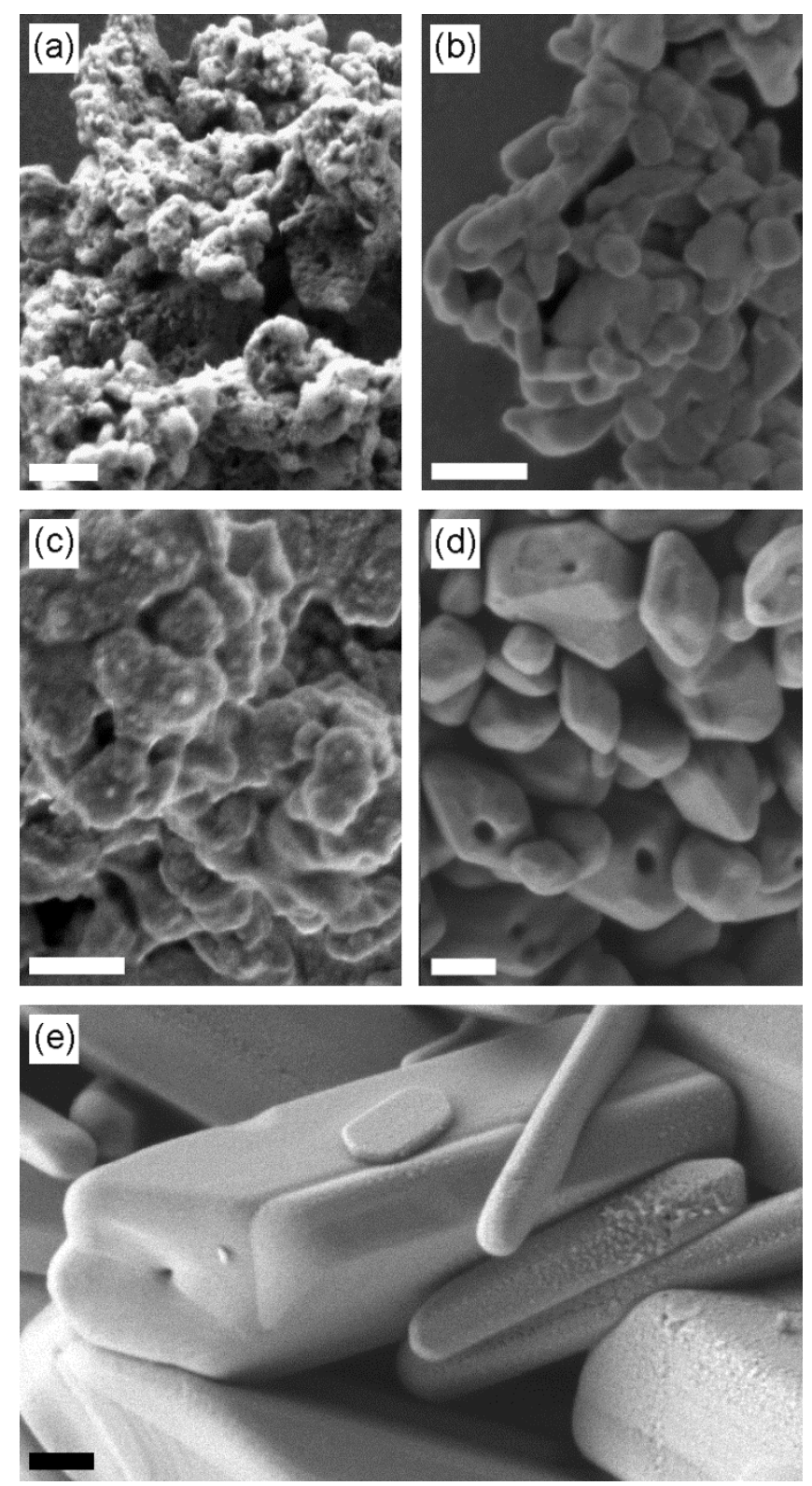

Fig. 2. SEM micrographs of $\mathrm{PbF}_{2}$ samples: (a) reference material; and products obtained with additives: (b) citric acid; (c) PACMA; (d) 12NH 2 ; and (e) AMPOH. Bar corresponds to $200 \mathrm{~nm}$. 


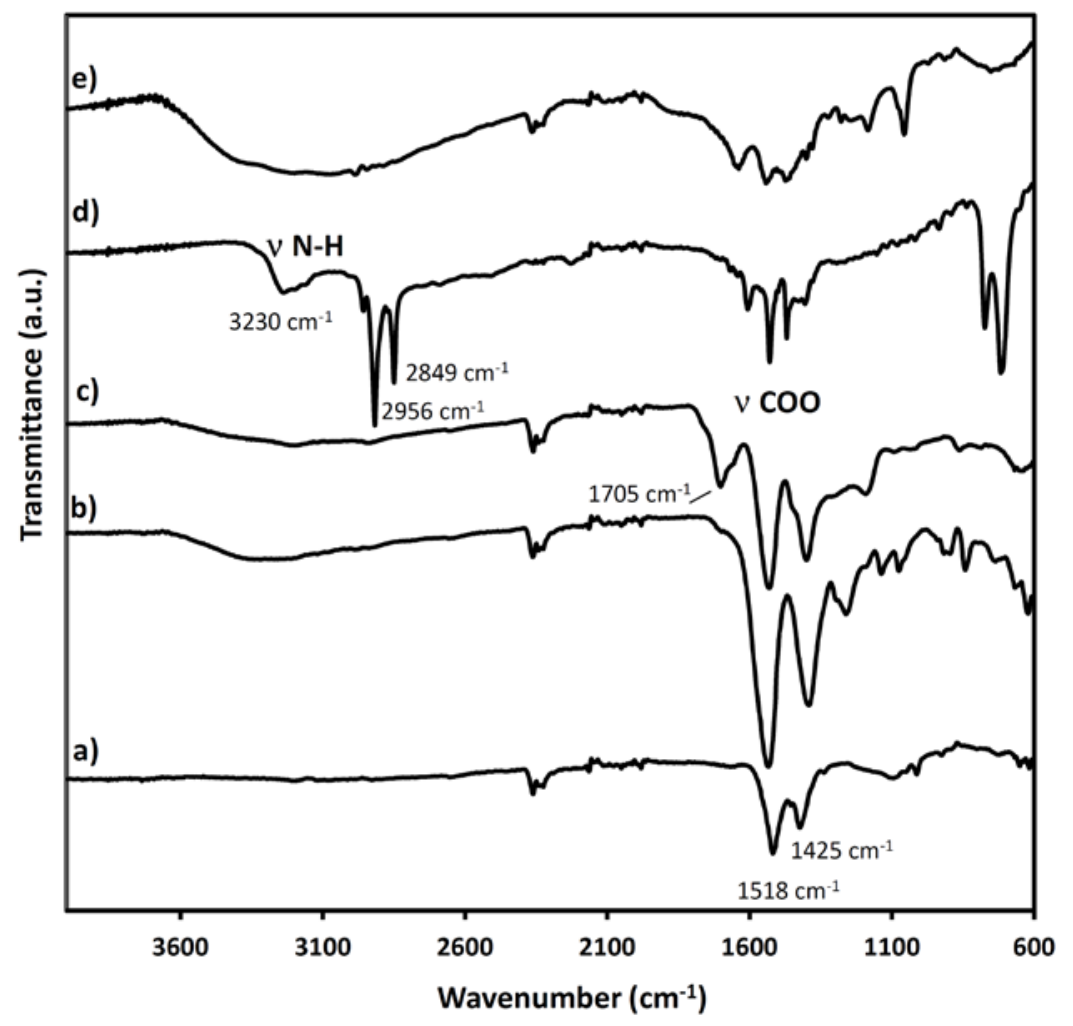

Fig. 3. ATR-FTIR spectrum of $\mathrm{PbF}_{2}$ samples: (a) reference material; and obtained with additives: (b) citric acid; (c) PACMA; (d) $12 \mathrm{NH}_{2}$; and (e) AMPOH. 